\title{
Determination of Water Quality Index and Suitability of Three Major Dams in Ekiti State, Nigeria
}

\author{
P. T. Olagbemide ${ }^{1,2 *}$, O. Owolabi1 \\ ${ }^{1}$ Department of Zoology, University of Ilorin, Ilorin; Nigeria \\ ${ }^{2}$ Department of Biological Sciences, AfeBabalola University, Ado-Ekiti, Nigeria \\ Email: ^Petseko2004@yahoo.co.uk
}

How to cite this paper: Olagbemide, P.T. and Owolabi, O. (2019) Determination of Water Quality Index and Suitability of Three Major Dams in Ekiti State, Nigeria. Open Access Library Journal, 6: e5719. https://doi.org/10.4236/oalib.1105719

Received: August 20, 2019

Accepted: September 6, 2019

Published: September 9, 2019

Copyright $\odot 2019$ by author(s) and Open Access Library Inc.

This work is licensed under the Creative Commons Attribution International License (CC BY 4.0).

http://creativecommons.org/licenses/by/4.0/

\begin{abstract}
Water Quality of Egbe, Ureje and Ero dams in Ekiti State was studied for a period of one year from November 2017 to October 2018. Twenty-four physicochemical parameters (temperature, conductivity, turbidity, $\mathrm{pH}, \mathrm{DO}_{2}$, BOD, COD, alkalinity, turbidity, total dissolved solids, hardness, colour, phosphates, nitrates, nitrite, $\mathrm{Cu}, \mathrm{Pb}, \mathrm{Mg}, \mathrm{K}, \mathrm{Mn}, \mathrm{Fe}, \mathrm{Na}, \mathrm{Ca}$ and $\mathrm{Zn}$ ) were considered in the computation of Water Quality Index (WQI) based on Weighted Arithmetic Water Quality Index Method. ANOVA was used to evaluate the significant differences between water quality variables at the dams. $T$-test was used to show the variations of physico-chemical parameters and WQI between the two seasons. WQI in Egbe dam ranges from 118.00164.67 (dry season) with a seasonal value of 145.69 and from $89.39-161.86$ (rainy season) with a seasonal value of 120.59. In Ureje dam, WQI ranges from 101.88 - 164.76 (dry season) with seasonal value of 134.08 and from 96.61 - 138.36 (rainy season) with a seasonal value of 123.39 while in Ero, WQI ranges from 32.28 - 45.13 (dry season) with a seasonal value of 39.43 and from 52.12 - 106.22 (rainy season) with a seasonal value of 81.84 . WQI of Egbe dam and Ureje dam during the dry and rainy seasons indicated poor water quality that is unfit for drinking while in Ero dam, the WQI during the dry season indicated good quality water while during the rainy season, the water in the dam was polluted. Our findings highlighted the deterioration of the dams due to anthropogenic activities and urbanization. There is an urgent need for proper management measures and suitable tools to restore the water quality of these dams. The formulas used to calculate the WQIs are easy to use thus providing a valuable tool for the accurate monitoring of water pollution in water bodies.
\end{abstract}




\section{Subject Areas}

Environmental Sciences

\section{Keywords}

Water Quality Index, Physico-Chemical Parameters, Anthropogenic Activities, Dams

\section{Introduction}

Water is the most critical resource for life and as a prime natural resource and precious national asset, it forms the chief constituent of ecosystem. In addition to the need of water for drinking, water resources play a vital role in various sectors of economy such as agriculture, livestock production, forestry, industrial activities, hydropower generation, fisheries and other creative activities. However, the aquatic environment is perceived to be at risk of several thousands of toxic chemicals, of both anthropogenic and natural origin. Environmental pollution especially in aquatic ecosystems is a current global environmental problem of the $21^{\text {st }}$ century due to limited supply of fresh water and the role of anthropogenic activities in deteriorating the water quality [1] [2]. Deterioration of the aquatic environment is associated with urbanization by human populations commencing with the industrial revolution in the eighteen century [3].

Dams in Ekiti State are surrounded by farmlands. A large amount of chemicals (fertilizers and pesticides) are used by farmers which can enter the dams through runoff, atmospheric deposition and leaching. Also, garbage and wastewaters are poured in the dams by inhabitants around the dams areas. All of these factors may lead to the contamination of Ekiti State waters. Reference [4] reported that surface water quality in a given location is largely influenced by both natural processes and by anthropogenic inputs. The deteriorating of water quality affects man, other animals and plant life with far-reaching consequences. It is therefore essential to prevent and control pollution of aquatic resources. Information on water quality and pollution sources is important for the implementation of sustainable water-use management strategies and to manage the environmental degradation, it is mandatory to assess the quality of the water sources from time to time [5] [6]. Thus, the evaluation of water quality is a critical element in the assessment of water resources.

Water quality of any specific area can be assessed using physical, chemical and biological parameters and these parameters are harmful for man when they occurred more than defined standard limits [7] [8]. This is because water quality characteristics of aquatic environment arise from a multitude of physical, chemical and biological interactions [9]. Some of these compounds are toxic to the ecosystem, some provide nutrients to aquatic organisms and others are responsible for the aesthetics of the water body [10]. One of the simplest methods to 
assess water quality conditions is by using water quality indices. Water Quality Index (WQI) provides a single number that expresses overall water quality at a particular location and time based on several water quality parameters. WQI incorporates data from multiple water quality parameters into a mathematical equation that rates the health status of a water body with number. Thus, WQI depicts the composite influence of different water quality parameters and communicates water quality information to the public and legislative decision makers [11]. The WQI was first formulated by Horton [12] in the United State and subsequently used by several workers for the quality assessment of different water resources in several places. Although WQI is not a new concept and is widely used in Nigeria, however its application as a method for water quality assessment is fairly undeveloped in Ekiti State and to the best of our knowledge, WQI of any of the Ekiti State water bodies has not been reported in the published literatures. Hence, the aim of this investigation was to determine the WQI for the three major water bodies in Ekiti State and provide baseline information on WQI on Ekiti State waters. This shall be helpful for the efficiency improvement in water quality management and policymaking on water resources in Ekiti State.

\section{Material and Methods}

\subsection{Study Area}

Ekiti state is located in the tropical climate with distinct wet and dry season [13]. Geographically, it is located between longitude $4^{\circ} 45^{\prime}$ to $5^{\circ} 45^{\prime}$ East of Greenwich Meridian and on latitude $7^{\circ} 15^{\prime}$ to $8^{\circ} 5^{\prime}$ North of the equator. It shares boundaries with Kwara State in the north (Figure 1), Kogi State in the north-east Osun State in the south and south-east. Ekiti state dams lie within the South western Basement Complex of Nigeria [14]. Human activities including defecation, washing, recreation (boating, swimming/bathing and fishing), agricultural activities and

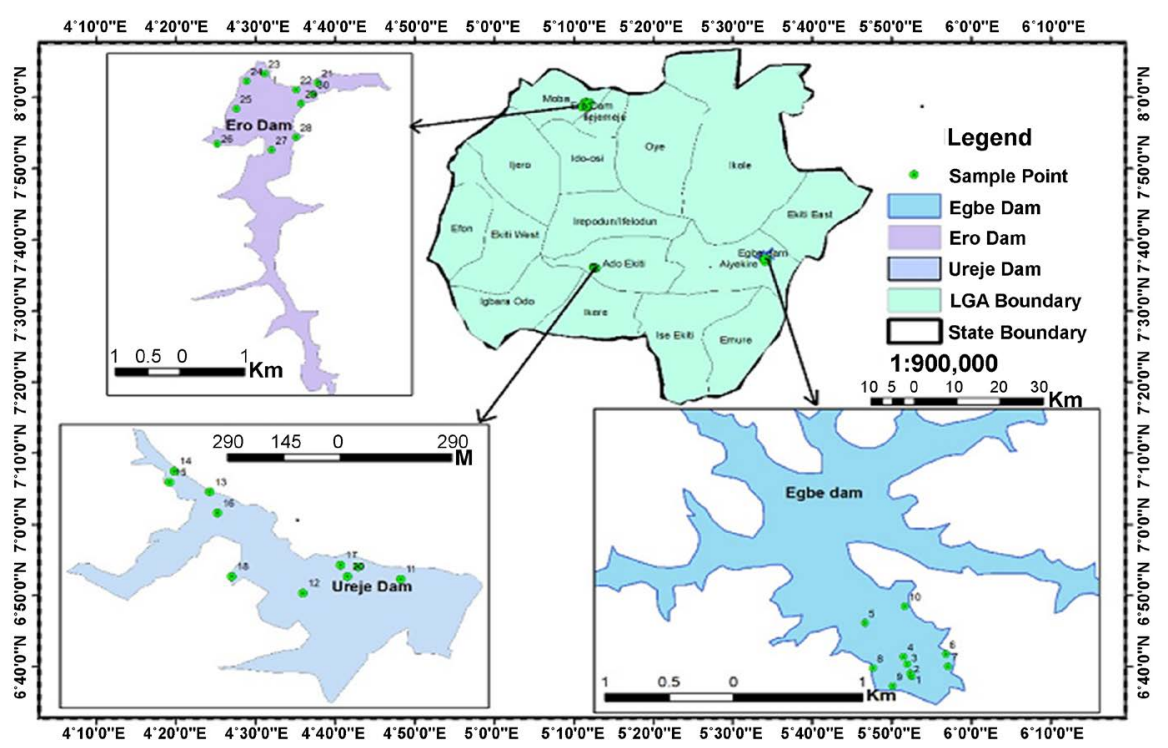

Figure 1. Map of Ekiti state dams showing the sampling sites. 
waste disposal are prevalent especially where human settlements exist around the dams. This study involves the three major dams in the state. They are: Ureje Dam, located at Longitude $5^{\circ} 14^{\prime} \mathrm{E}$ and Latitude $7^{\circ} 38.2^{\prime} \mathrm{N}$; Egbe Dam, situated at Longitude $5^{\circ} 36.9^{\prime} \mathrm{E}$ and Latitude $7^{\circ} 37.1^{\prime} \mathrm{N}$ and Ero Dam, situated at Ikun-Ekiti, Moba Local Government Area of Ekiti State, Nigeria between latitudes $7^{\circ} 15^{\prime}$ and $8^{\circ} 5^{\prime} \mathrm{N}$ and longitude $4^{\circ} 45^{\prime}$ and $5^{\circ} 31^{\prime} \mathrm{E}$.

\subsection{Sample Collection}

Samples of surface water were collected monthly from Ureje, Ero and Egbe dams from November, 2017 to October, 2018. Ten sampling sites were chosen in each dam to make pooled water sample monthly according to [15] [16] [17]. The sites were selected to represent the open water bodies and different variations within each dam so as to obtain a realistic overall representation. The GPS of sampling sites were taken using Garmin hand-held GPS. The geographical location of each selected sampling site within the reservoir is shown in Table 1.

Water samples from all ten sampling sites were collected during morning hours between 8.00 to 10.00 a.m. at a depth of about $0.3 \mathrm{~m}$ below water surface into 2 Litre polyethylene plastic containers. Prior to sampling, the containers were cleaned with $10 \%$ nitric acid and rinsed with distilled water. The containers were rinsed three times with the dam water at the time of sampling. Samples were then collected by direct immersion of the sampling containers into the dam. All sample containers were properly labeled to indicate date of sampling and the sampling location. Immediately after sample collection, $2 \mathrm{ml}$ of concentrated nitric acid was added to the water samples for metal analysis to reduce adsorption of metals onto the walls of the plastic containers and to prevent microbial growth in the water sample. Winkler's bottles were used to collect water samples for COD and BOD. Samples were transported to the laboratory at about $4^{\circ} \mathrm{C}$ (ice/cold packs) to retard reactions and stored at $4^{\circ} \mathrm{C}$ awaiting analysis. In

Table 1. The sampling sites and GPS co-ordinates.

\begin{tabular}{|c|c|c|c|c|c|c|c|c|}
\hline \multicolumn{3}{|c|}{ EGBE DAM } & \multicolumn{3}{|c|}{ UREJE DAM } & \multicolumn{3}{|c|}{ ERO DAM } \\
\hline $\mathrm{S} / \mathrm{N}$ & Lat. & Long. & $\mathrm{S} / \mathrm{N}$ & Lat. & Long. & $\mathrm{S} / \mathrm{N}$ & Lat. & Long. \\
\hline 1 & $7^{\circ} 38^{\prime} 0.15^{\prime \prime} \mathrm{N}$ & $5^{\circ} 34^{\prime} 6.15^{\prime \prime} \mathrm{E}$ & 11 & $7^{\circ} 36^{\prime} 0.30^{\prime \prime N}$ & $5^{\circ} 12^{\prime} 52.98^{\prime \prime} \mathrm{E}$ & 21 & $7^{\circ} 59^{\prime} 14.68^{\prime \prime N}$ & $5^{\circ} 12^{\prime} 11.57^{\prime \prime} \mathrm{E}$ \\
\hline 2 & $7^{\circ} 37^{\prime} 49.93^{\prime \prime} \mathrm{N}$ & $5^{\circ} 33^{\prime} 44.19^{\prime \prime} \mathrm{E}$ & 12 & $7^{\circ} 35^{\prime} 58.97^{\prime \prime} \mathrm{N}$ & $5^{\circ} 12^{\prime} 44.77^{\prime \prime E}$ & 22 & $7^{\circ} 59^{\prime} 1.24 " \mathrm{~N}$ & $5^{\circ} 11^{\prime} 49.96 " \mathrm{E}$ \\
\hline 3 & $7^{\circ} 37^{\prime} 39.96 " \mathrm{~N}$ & $5^{\circ} 33^{\prime} 42.62^{\prime \prime} \mathrm{E}$ & 13 & $7^{\circ} 36^{\prime} 8.46^{\prime \prime} \mathrm{N}$ & $5^{\circ} 12^{\prime} 36.96^{\prime \prime} \mathrm{E}$ & 23 & $7^{\circ} 59^{\prime} 19.58^{\prime \prime N}$ & $5^{\circ} 11^{\prime} 38.59^{\prime \prime} \mathrm{E}$ \\
\hline 4 & $7^{\circ} 37^{\prime} 12.77^{\prime \prime} \mathrm{N}$ & $5^{\circ} 34^{\prime} 12.02^{\prime \prime} \mathrm{E}$ & 14 & $7^{\circ} 36^{\prime} 10.41 " \mathrm{~N}$ & $5^{\circ} 12^{\prime} 33.99^{\prime \prime E}$ & 24 & $7^{\circ} 59^{\prime} 15.42 " \mathrm{~N}$ & $5^{\circ} 11^{\prime} 29.52^{\prime \prime} \mathrm{E}$ \\
\hline 5 & $7^{\circ} 37^{\prime} 16.20^{\prime \prime} \mathrm{N}$ & $5^{\circ} 34^{\prime} 3.00 " \mathrm{E}$ & 15 & $7^{\circ} 36^{\prime} 9.36^{\prime \prime N}$ & $5^{\circ} 12^{\prime} 33.60^{\prime \prime} \mathrm{E}$ & 25 & $7^{\circ} 59^{\prime} 0.51 " \mathrm{~N}$ & $5^{\circ} 11^{\prime} 35.96^{\prime \prime} \mathrm{E}$ \\
\hline 6 & $7^{\circ} 37^{\prime} 35.25^{\prime \prime} \mathrm{N}$ & $5^{\circ} 34^{\prime} 23.55^{\prime \prime} \mathrm{E}$ & 16 & $7^{\circ} 36^{\prime} 6.48^{\prime \prime N}$ & $5^{\circ} 12^{\prime} 37.62^{\prime \prime E}$ & 26 & $7^{\circ} 58^{\prime} 46.31 " \mathrm{~N}$ & $5^{\circ} 11^{\prime} 22.93^{\prime \prime} \mathrm{E}$ \\
\hline 7 & $7^{\circ} 37^{\prime} 4.02 " \mathrm{~N}$ & $5^{\circ} 34^{\prime} 23.93^{\prime \prime} \mathrm{E}$ & 17 & $7^{\circ} 36^{\prime} 1.61^{\prime \prime N}$ & $5^{\circ} 12^{\prime} 47.92^{\prime \prime E}$ & 27 & $7^{\circ} 58^{\prime} 14.84 " \mathrm{~N}$ & $5^{\circ} 11^{\prime} 32.92^{\prime \prime} \mathrm{E}$ \\
\hline 8 & $7^{\circ} 37^{\prime} 28.09 " \mathrm{~N}$ & $5^{\circ} 33^{\prime} 53.02^{\prime \prime} \mathrm{E}$ & 18 & $7^{\circ} 36^{\prime} 0.54^{\prime \prime} \mathrm{N}$ & $5^{\circ} 12^{\prime} 38.81^{\prime \prime E}$ & 28 & $7^{\circ} 58^{\prime} 32.51 " \mathrm{~N}$ & $5^{\circ} 11^{\prime} 44.57^{\prime \prime} \mathrm{E}$ \\
\hline 9 & $7^{\circ} 37^{\prime} 1.91 " \mathrm{~N}$ & $5^{\circ} 34^{\prime} 12.11^{\prime \prime E}$ & 19 & $7^{\circ} 36^{\prime} 1.45^{\prime \prime} \mathrm{N}$ & $5^{\circ} 12^{\prime} 49.42^{\prime \prime} \mathrm{E}$ & 29 & $7^{\circ} 58^{\prime} 50.92 " \mathrm{~N}$ & $5^{\circ} 11^{\prime} 42.88^{\prime \prime} \mathrm{E}$ \\
\hline 10 & $7^{\circ} 37^{\prime} 49.16^{\prime \prime} \mathrm{N}$ & $5^{\circ} 33^{\prime} 55.76^{\prime \prime} \mathrm{E}$ & 20 & $7^{\circ} 36^{\prime} 0.55^{\prime \prime} \mathrm{N}$ & $5^{\circ} 12^{\prime} 48.51 " \mathrm{E}$ & 30 & $7^{\circ} 57^{\prime} 45.39^{\prime \prime} \mathrm{N}$ & $5^{\circ} 11^{\prime} 39.13^{\prime \prime} \mathrm{E}$ \\
\hline
\end{tabular}


situ water quality variable were recorded using Jenway portable $970 \mathrm{DO}_{2}$ Meter and Jenway $430 \mathrm{pH} /$ Conductivity Meter. The in situ water quality parameters taken were temperature $\left({ }^{\circ} \mathrm{C}\right), \mathrm{pH}$ and electrical conductivity $(\mu \mathrm{S} / \mathrm{cm})$ and dissolved oxygen $(\mathrm{mg} / \mathrm{L})$.

\subsection{Water Sample Analysis}

The samples were analyzed in the laboratory as per standard methods [18], for different Physico-Chemical parameters namely, conductivity (EC), turbidity (Tur), Total solids (TS), $\mathrm{pH}, \mathrm{DO}_{2}, \mathrm{BOD}, \mathrm{COD}$, Total alkalinity (Totalk), total dissolved solids (TDS), Total hardness (TH), colour (Col), $\mathrm{PO}_{4}, \mathrm{NO}_{3}$ and $\mathrm{NO}_{2}$. Metals $(\mathrm{Cu}, \mathrm{Pb}, \mathrm{Mg}, \mathrm{K}, \mathrm{Mn}, \mathrm{Fe}, \mathrm{Na}, \mathrm{Ca}, \mathrm{Zn}$ and $\mathrm{Cd})$ in the water samples were determined by atomic absorption spectrophotometric method using Atomic absorption spectrophotometer (Bulk Scientific Model 211 VGP) [19].

\subsection{Data Analysis}

Statistical analysis was performed using SPSS software version 23 and Excel spreadsheet. The means of the physico-chemical variables, evaluation of significant differences between different samples were determined using descriptive statistics and analysis of variance (ANOVA) respectively. Results of the test were considered significant if the calculated $\mathrm{P}$ values were $\leq 0.05$ or $\leq 0.01$. $T$-test was used to show the variations of physico-chemical parameters between the two seasons.

Twenty-four physico-chemical parameters were used for calculation of Water Quality index for each of the dams using weighted arithmetic index method [20] according to the following equations:

$$
k=\frac{1}{\sum_{i=1}^{n} 1 / S_{i}}
$$

in which $k=$ constant of proportionality

$S_{i}=$ permissible limit for the $i$ th parameter

$n=$ number of parameters.

$$
\begin{gathered}
\text { Relative Weight }\left(W_{i}\right)=\frac{k}{S_{i}} \\
\text { Quality Rating Scale }\left(Q_{i}\right)=100\left[\left(V_{i}-V_{o} / S_{i}-V_{o}\right)\right]
\end{gathered}
$$

where, $V_{i}$ is estimated concentration of $i$ th parameter in the analyzed water

$V_{o}$ is the ideal value of this parameter in pure water

$V_{o}=0$ (except $\mathrm{pH}=7.0$ and $\left.\mathrm{DO}=14.6 \mathrm{mg} / \mathrm{l}\right)$

$S_{i}$ is recommended standard value of ith parameter.

Water Quality Index (WQI) is then calculated from the following equation

$$
\mathrm{WQI}=\frac{\sum_{i=1}^{n} Q_{i} W_{i}}{\sum_{i=1}^{n} W_{i}}
$$

The standards for the drinking water recommended by the Indian Council of 
Medical Research (ICMR), World Health Organization (WHO), Nigerian Industrial Standard (NIS), National Environmental Standards and Regulations Enforcement Agency (NESREA) and Bureau of Indian standards (BIS) were considered for the computation of quality rating $\left(Q_{i}\right)$ and unit weights $\left(W_{i}\right)$. The water quality was classified as excellent (if the WQI value is 0 to 25 ), good (26 to 50 ), moderately polluted (51 to 75 ), severely polluted (76 to 100), and unfit for drinking (above 100) according to [21] and [22].

\section{Results and Discussion}

Average values for each water quality parameter per season in the three dams used in the computation of water quality index are presented in Table 2. The

Table 2. Seasonal variations of the physicochemical parameters of the dams.

\begin{tabular}{|c|c|c|c|c|c|c|c|c|}
\hline \multirow{3}{*}{ PARAMETERS } & \multicolumn{6}{|c|}{ YEAR 2017-2018 } & \multirow[b]{3}{*}{$\begin{array}{l}\text { STANDARD } \\
\text { VALUE }\end{array}$} & \multirow[b]{3}{*}{ AGENCIES } \\
\hline & \multicolumn{2}{|c|}{ EGBE DAM } & \multicolumn{2}{|c|}{ UREJE DAM } & \multicolumn{2}{|c|}{ ERO DAM } & & \\
\hline & DRY SEASON & $\begin{array}{l}\text { RAINY } \\
\text { SEASON }\end{array}$ & DRY SEASON & $\begin{array}{l}\text { RAINY } \\
\text { SEASON }\end{array}$ & DRY SEASON & $\begin{array}{l}\text { RAINY } \\
\text { SEASON }\end{array}$ & & \\
\hline $\mathrm{T}\left({ }^{\circ} \mathrm{C}\right)$ & $27.93 \pm 3.35^{\mathrm{a}}$ & $26.61 \pm 0.50^{\mathrm{a}}$ & $27.44 \pm 3.01^{\mathrm{a}}$ & $25.66 \pm 0.87^{\mathrm{a}}$ & $26.75 \pm 2.35^{\mathrm{a}}$ & $26.14 \pm 0.97^{\mathrm{a}}$ & $<40$ & WHO \\
\hline $\mathrm{pH}$ & $8.30 \pm 1.55^{\mathrm{a}}$ & $8.83 \pm 1.30^{\mathrm{a}}$ & $8.11 \pm 0.96^{\mathrm{a}}$ & $8.72 \pm 1.39^{\mathrm{a}}$ & $9.05 \pm 0.54^{\mathrm{a}}$ & $9.07 \pm 1.33^{\mathrm{a}}$ & 8.5 & ICMB/BIS \\
\hline $\mathrm{DO}_{2}(\mathrm{mg} / \mathrm{l})$ & $7.77 \pm 2.85^{\mathrm{a}}$ & $9.06 \pm 3.90^{\mathrm{a}}$ & $6.86 \pm 1.85^{\mathrm{a}}$ & $7.65 \pm 2.14^{\mathrm{a}}$ & $8.85 \pm 3.13^{\mathrm{a}}$ & $10.07 \pm 1.22^{\mathrm{a}}$ & 5 & ICMB/BIS \\
\hline TUR (NTU) & $2.37 \pm 1.31^{\mathrm{a}}$ & $2.91 \pm 0.39^{\mathrm{a}}$ & $2.78 \pm 0.89^{\mathrm{a}}$ & $3.43 \pm 1.01^{\mathrm{a}}$ & $2.76 \pm 1.75^{\mathrm{a}}$ & $2.39 \pm 1.00^{\mathrm{a}}$ & 5 & WHO \\
\hline COL (Hazen) & $5.00 \pm 0.00^{\mathrm{a}}$ & $5.00 \pm 0.00^{\mathrm{a}}$ & $5.42 \pm 1.02^{\mathrm{a}}$ & $7.29 \pm 2.55^{\mathrm{a}}$ & $5.00 \pm 0.00^{\mathrm{a}}$ & $5.00 \pm 0.00^{\mathrm{a}}$ & 5 & BIS \\
\hline $\mathrm{EC}(\mu \mathrm{S} / \mathrm{cm})$ & $126.08 \pm 29.08^{\mathrm{a}}$ & $134.22 \pm 18.91^{\mathrm{a}}$ & $202.56 \pm 74.04^{\mathrm{a}}$ & $212.65 \pm 57.09^{\mathrm{a}}$ & $124.93 \pm 10.67^{\mathrm{a}}$ & $129.67 \pm 36.08^{a}$ & 300 & ICMB \\
\hline $\mathrm{TS}(\mathrm{mg} / \mathrm{l})$ & $50.43 \pm 11.98^{\mathrm{a}}$ & $72.09 \pm 11.80^{\mathrm{b}}$ & $94.97 \pm 36.37^{\mathrm{a}}$ & $99.53 \pm 22.20^{\mathrm{a}}$ & $58.34 \pm 9.56^{\mathrm{a}}$ & $63.77 \pm 20.74^{\mathrm{a}}$ & - & - \\
\hline TDS (mg/l) & $47.40 \pm 10.63^{\mathrm{a}}$ & $67.43 \pm 10.08^{b}$ & $87.26 \pm 34.78^{\mathrm{a}}$ & $92.62 \pm 19.15^{\mathrm{a}}$ & $52.17 \pm 9.66^{\mathrm{a}}$ & $57.42 \pm 20.12^{\mathrm{a}}$ & 500 & ICMB/BIS \\
\hline TSS (mg/l) & $3.26 \pm 1.20^{\mathrm{a}}$ & $4.67 \pm 1.04^{\mathrm{a}}$ & $7.72 \pm 2.46^{\mathrm{a}}$ & $6.98 \pm 3.12^{\mathrm{a}}$ & $6.15 \pm 1.31^{\mathrm{a}}$ & $6.35 \pm 0.82^{\mathrm{a}}$ & 25 & NESREA \\
\hline $\mathrm{TH}(\mathrm{mg} / \mathrm{l})$ & $63.88 \pm 4.69^{\mathrm{a}}$ & $66.31 \pm 8.90^{\mathrm{a}}$ & $97.05 \pm 17.08^{\mathrm{a}}$ & $86.32 \pm 19.26^{\mathrm{a}}$ & $76.07 \pm 6.92^{\mathrm{a}}$ & $75.34 \pm 7.54^{\mathrm{a}}$ & 300 & ICMB/BIS \\
\hline Totalk (mg/l) & $33.82 \pm 8.02^{\mathrm{a}}$ & $33.40 \pm 6.47^{\mathrm{a}}$ & $53.60 \pm 16.70^{\mathrm{a}}$ & $47.41 \pm 8.83^{\mathrm{a}}$ & $46.10 \pm 7.63^{\mathrm{a}}$ & $36.61 \pm 11.49^{\mathrm{a}}$ & 120 & ICMB \\
\hline BOD (mg/l) & $5.68 \pm 1.01^{\mathrm{a}}$ & $5.21 \pm 0.59^{\mathrm{a}}$ & $7.46 \pm 1.67^{\mathrm{a}}$ & $6.32 \pm 0.70^{\mathrm{a}}$ & $5.21 \pm 0.53^{\mathrm{a}}$ & $5.54 \pm 0.84^{\mathrm{a}}$ & 5 & ICMB/BIS \\
\hline COD (mg/l) & $10.36 \pm 2.53^{\mathrm{a}}$ & $6.99 \pm 0.73^{b}$ & $11.80 \pm 1.88^{\mathrm{a}}$ & $9.53 \pm 1.11^{\mathrm{b}}$ & $8.62 \pm 0.94^{\mathrm{a}}$ & $9.13 \pm 1.32^{\mathrm{a}}$ & 10 & WHO \\
\hline $\mathrm{NO}_{3}(\mathrm{mg} / \mathrm{l})$ & $0.041 \pm 0.04^{\mathrm{a}}$ & $\mathrm{ND}^{\mathrm{a}}$ & $0.143 \pm 0.22^{\mathrm{a}}$ & $0.20 \pm 0.27^{\mathrm{a}}$ & $0.187 \pm 0.27^{\mathrm{a}}$ & $0.56 \pm 0.56^{\mathrm{a}}$ & 45 & 1CMB/BIS \\
\hline $\mathrm{NO}_{2}(\mathrm{mg} / \mathrm{l})$ & $0.006 \pm 0.01^{\mathrm{a}}$ & $\mathrm{ND}^{\mathrm{a}}$ & $0.033 \pm 0.07^{\mathrm{a}}$ & $\mathrm{ND}^{\mathrm{a}}$ & $0.002 \pm 0.002^{\mathrm{a}}$ & $\mathrm{ND}^{\mathrm{a}}$ & 0.2 & NIS \\
\hline $\mathrm{PO}_{4}(\mathrm{mg} / \mathrm{l})$ & $0.015 \pm 0.02^{\mathrm{a}}$ & $\mathrm{ND}^{\mathrm{a}}$ & $0.024 \pm 0.02^{\mathrm{a}}$ & $\mathrm{ND}^{\mathrm{a}}$ & $0.002 \pm 0.003^{\mathrm{a}}$ & $\mathrm{ND}^{\mathrm{a}}$ & 0.1 & USEPA \\
\hline $\mathrm{Na}(\mathrm{ppm})$ & $22.62 \pm 7.39^{\mathrm{a}}$ & $22.94 \pm 5.99^{\mathrm{a}}$ & $10.74 \pm 2.09^{\mathrm{a}}$ & $22.39 \pm 6.09^{b}$ & $40.22 \pm 1.29^{\mathrm{a}}$ & $53.90 \pm 3.66^{\mathrm{b}}$ & 200 & NIS \\
\hline $\mathrm{K}(\mathrm{ppm})$ & $11.11 \pm 1.61^{\mathrm{a}}$ & $13.16 \pm 3.44^{\mathrm{b}}$ & $11.60 \pm 2.00^{\mathrm{a}}$ & $17.50 \pm 3.12^{\mathrm{b}}$ & $31.57 \pm 1.09^{\mathrm{a}}$ & $26.11 \pm 2.22^{\mathrm{a}}$ & 10 & WHO \\
\hline $\mathrm{Fe}(\mathrm{ppm})$ & $0.18 \pm 0.02^{\mathrm{a}}$ & $0.10 \pm 0.03^{b}$ & $0.31 \pm 0.04^{\mathrm{a}}$ & $0.20 \pm 0.04^{\mathrm{b}}$ & $0.06 \pm 0.005^{\mathrm{a}}$ & $0.081 \pm 0.03^{\mathrm{b}}$ & 0.1 & WHO \\
\hline Mn (ppm) & $0.21 \pm 0.05^{\mathrm{a}}$ & $0.17 \pm 0.04^{\mathrm{b}}$ & $0.19 \pm 0.08^{\mathrm{a}}$ & $0.12 \pm 0.04^{\mathrm{a}}$ & $0.096 \pm 0.005^{\mathrm{a}}$ & $0.24 \pm 0.02^{\mathrm{a}}$ & 0.1 & BIS \\
\hline $\mathrm{Zn}(\mathrm{ppm})$ & $0.24 \pm 0.07^{\mathrm{a}}$ & $0.27 \pm 0.10^{\mathrm{a}}$ & $0.12 \pm 0.005^{\mathrm{a}}$ & $0.30 \pm 0.09^{\mathrm{b}}$ & $0.101 \pm 0.002^{\mathrm{a}}$ & $0.39 \pm 0.034^{\mathrm{b}}$ & 3.0 & NIS \\
\hline $\mathrm{Mg}(\mathrm{ppm})$ & $4.82 \pm 0.44^{\mathrm{a}}$ & $4.87 \pm 0.69^{\mathrm{a}}$ & $4.21 \pm 0.34^{\mathrm{a}}$ & $4.96 \pm 0.53^{\mathrm{b}}$ & $2.25 \pm 0.05^{\mathrm{a}}$ & $5.57 \pm 0.31^{\mathrm{b}}$ & 30 & BIS \\
\hline $\mathrm{Cu}(\mathrm{ppm})$ & $0.17 \pm 0.04^{\mathrm{a}}$ & $0.16 \pm 0.05^{\mathrm{a}}$ & $0.12 \pm 0.03^{\mathrm{a}}$ & $0.16 \pm 0.03^{b}$ & $0.030 \pm 0.004^{\mathrm{a}}$ & $0.069 \pm 0.012^{\mathrm{b}}$ & 0.05 & BIS \\
\hline $\mathrm{Pb}(\mathrm{ppm})$ & $0.005 \pm 0.003^{\mathrm{a}}$ & $0.0013 \pm 0.0010^{b}$ & $0.001 \pm 0.0004^{\mathrm{a}}$ & $0.001 \pm 0.0005^{\mathrm{a}}$ & $0.0031 \pm 0.0006^{\mathrm{a}}$ & $0.0048 \pm 0.0014^{\mathrm{a}}$ & 0.05 & WHO \\
\hline
\end{tabular}

Physico-chemical parameter values of dry season and wet season with similar superscription are not significant ${ }^{\mathrm{a}}$. ND—Not detected ${ }^{\mathrm{b}}$. 
average values of TS, TDS, COD, $\mathrm{K}, \mathrm{Fe}$ and $\mathrm{Mn}$ were significantly different $(\mathrm{P} \geq$ 0.05 ) between dry and rainy seasons in Egbe dam. However, average $\mathrm{pH}$, turbidity, $\mathrm{DO}_{2}$, EC, TS, TDS, TSS, TH, Na, K, $\mathrm{Zn}$ and $\mathrm{Mg}$ were higher in the wet season compared to the dry season in Egbe dam. In Ureje dam, the differences between dry season and rainy season values of $\mathrm{COD}, \mathrm{Na}, \mathrm{Ca}, \mathrm{K}, \mathrm{Fe}, \mathrm{Zn}$ and $\mathrm{Cu}$ were significant $(\mathrm{P} \geq 0.05)$. But in the dam, the average values of $\mathrm{pH}$, turbidity, $\mathrm{DO}_{2}$, colour, EC, TS, TDS, $\mathrm{NO}_{3}, \mathrm{Na}, \mathrm{Ca}, \mathrm{K}, \mathrm{Zn}, \mathrm{Mg}$ and $\mathrm{Cu}$ were higher during the rainy season than the dry season. In Ero dam, only $\mathrm{Na}, \mathrm{Fe}, \mathrm{Zn}, \mathrm{Mg}$ and $\mathrm{Cu}$ showed significant difference $(P \geq 0.05)$ between the dry season and rainy season. However, $\mathrm{pH}, \mathrm{DO}_{2}, \mathrm{EC}, \mathrm{TS}$, TDS, TSS, $\mathrm{BOD}, \mathrm{COD}, \mathrm{NO}_{3}, \mathrm{Fe}, \mathrm{Zn}, \mathrm{Mg}, \mathrm{Cu}$ and $\mathrm{Pb}$ showed higher values during the rainy season than dry season. The water sample analysis reveals that dams' water is not fit for drinking with respect to $\mathrm{pH}, \mathrm{DO}_{2}$, $\mathrm{K}, \mathrm{BOD}, \mathrm{COD}$ (except in Ero dam), Fe (except in Ero dam), Zn (except in Egbe dam), $\mathrm{Cu}$ and colour (only Ureje dam), the concentrations of these parameters exceed the permissible limits of WHO and other international organizations. Table 3 and Table 4 show the seasonal WQI for Egbe dam during the dry and rainy seasons respectively. Table 5 and Table 6 show the seasonal WQI for Ureje dam for dry and rainy seasons respectively while Table 7 and Table 8

Table 3. Water quality index in Egbe dam during the dry season.

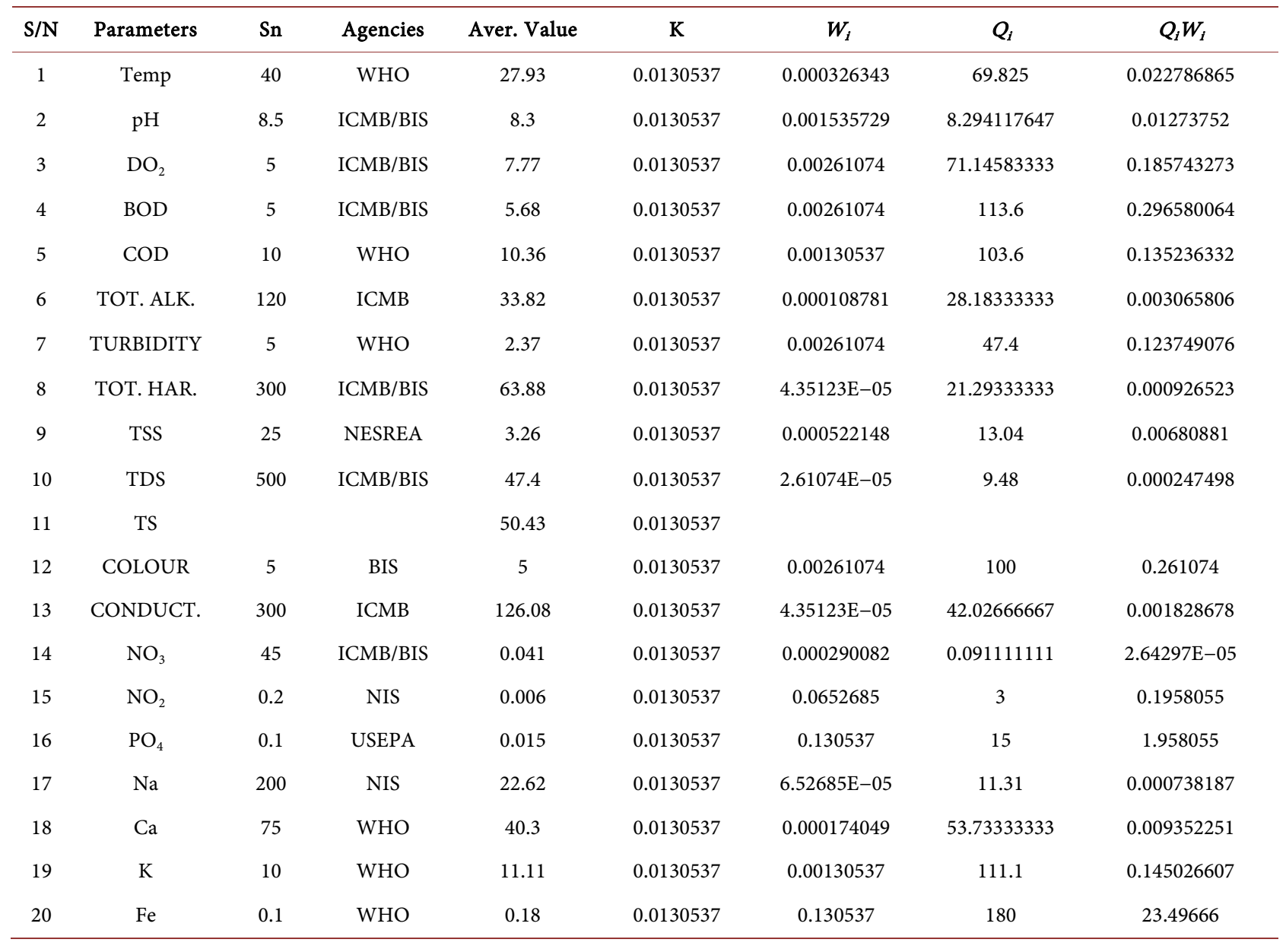




\section{Continued}

\begin{tabular}{|c|c|c|c|c|c|c|c|c|}
\hline 21 & $\mathrm{Mn}$ & 0.1 & BIS & 0.21 & 0.0130537 & 0.130537 & 210 & 27.41277 \\
\hline 22 & $\mathrm{Zn}$ & 3 & NIS & 0.24 & 0.0130537 & 0.004351233 & 8 & 0.034809867 \\
\hline 23 & $\mathrm{Mg}$ & 30 & BIS & 4.82 & 0.0130537 & 0.000435123 & 16.06666667 & 0.006990982 \\
\hline 24 & $\mathrm{Cu}$ & 0.05 & BIS & 0.17 & 0.0130537 & 0.261074 & 340 & 88.76516 \\
\hline \multirow[t]{3}{*}{25} & $\mathrm{~Pb}$ & 0.05 & WHO & 0.005 & 0.0130537 & 0.261074 & 10 & 2.61074 \\
\hline & & & & & $\sum W_{i}=$ & 1.00000309 & $\sum Q_{i} W_{i}=$ & 145.6869193 \\
\hline & & & & & & $\sum Q_{i} W_{i} / \sum W_{i}$ & $=145.69$ & \\
\hline
\end{tabular}

Table 4. Water quality index in Egbe dam during rainy season.

\begin{tabular}{|c|c|c|c|c|c|c|c|c|}
\hline $\mathrm{S} / \mathrm{N}$ & Parameters & Sn & Agencies & Aver. Value & $\mathrm{K}$ & $W_{i}$ & $Q_{i}$ & $Q_{i} W_{i}$ \\
\hline 1 & Temp & 40 & WHO & 26.61 & 0.0130537 & 0.000326343 & 66.525 & 0.021709935 \\
\hline 2 & $\mathrm{pH}$ & 8.5 & ICMB/BIS & 8.83 & 0.0130537 & 0.001535729 & 14.52941176 & 0.022313245 \\
\hline 3 & $\mathrm{DO}_{2}$ & 5 & ICMB/BIS & 9.06 & 0.0130537 & 0.00261074 & 57.70833333 & 0.150661454 \\
\hline 4 & BOD & 5 & ICMB/BIS & 5.21 & 0.0130537 & 0.00261074 & 104.2 & 0.272039108 \\
\hline 5 & COD & 10 & WHO & 6.99 & 0.0130537 & 0.00130537 & 69.9 & 0.091245363 \\
\hline 6 & TOT. ALK. & 120 & ICMB & 33.4 & 0.0130537 & 0.000108781 & 27.83333333 & 0.003027733 \\
\hline 7 & TURBIDITY & 5 & WHO & 2.91 & 0.0130537 & 0.00261074 & 58.2 & 0.151945068 \\
\hline 8 & TOT. HAR. & 300 & ICMB/BIS & 66.31 & 0.0130537 & $4.35123 \mathrm{E}-05$ & 22.10333333 & 0.000961768 \\
\hline 9 & TSS & 25 & NESREA & 4.67 & 0.0130537 & 0.000522148 & 18.68 & 0.009753725 \\
\hline 10 & TDS & 500 & ICMB/BIS & 67.43 & 0.0130537 & $2.61074 \mathrm{E}-05$ & 13.486 & 0.000352084 \\
\hline 11 & TS & & & 72.09 & 0.0130537 & & & \\
\hline 12 & COLOUR & 5 & BIS & 5 & 0.0130537 & 0.00261074 & 100 & 0.261074 \\
\hline 13 & CONDUCT. & 300 & ICMB & 134.22 & 0.0130537 & $4.35123 \mathrm{E}-05$ & 44.74 & 0.001946742 \\
\hline 14 & $\mathrm{NO}_{3}$ & 45 & ICMB/BIS & 0 & 0.0130537 & 0.000290082 & 0 & 0 \\
\hline 15 & $\mathrm{NO}_{2}$ & 0.2 & NIS & 0 & 0.0130537 & 0.0652685 & 0 & 0 \\
\hline 16 & $\mathrm{PO}_{4}$ & 0.1 & USEPA & 0 & 0.0130537 & 0.130537 & 0 & 0 \\
\hline 17 & $\mathrm{Na}$ & 200 & NIS & 22.94 & 0.0130537 & $6.52685 \mathrm{E}-05$ & 11.47 & 0.00074863 \\
\hline 18 & $\mathrm{Ca}$ & 75 & WHO & 54.92 & 0.0130537 & 0.000174049 & 73.22666667 & 0.012745053 \\
\hline 19 & $\mathrm{~K}$ & 10 & WHO & 13.16 & 0.0130537 & 0.00130537 & 131.6 & 0.171786692 \\
\hline 20 & $\mathrm{Fe}$ & 0.1 & WHO & 0.1 & 0.0130537 & 0.130537 & 100 & 13.0537 \\
\hline 21 & $\mathrm{Mn}$ & 0.1 & BIS & 0.17 & 0.0130537 & 0.130537 & 170 & 22.19129 \\
\hline 22 & $\mathrm{Zn}$ & 3 & NIS & 0.27 & 0.0130537 & 0.004351233 & 9 & 0.0391611 \\
\hline 23 & $\mathrm{Mg}$ & 30 & BIS & 4.87 & 0.0130537 & 0.000435123 & 16.23333333 & 0.007063502 \\
\hline 24 & $\mathrm{Cu}$ & 0.05 & BIS & 0.16 & 0.0130537 & 0.261074 & 320 & 83.54368 \\
\hline \multirow[t]{3}{*}{25} & $\mathrm{~Pb}$ & 0.05 & WHO & 0.0013 & 0.0130537 & 0.261074 & 2.6 & 0.6787924 \\
\hline & & & & & $\sum W_{i}=$ & 1.00000309 & $\sum Q_{i} W_{i}=$ & 120.6859976 \\
\hline & & & & & & $\sum Q_{i} W_{i} / \sum W_{i}$ & $=120.69$ & \\
\hline
\end{tabular}


Table 5. Water quality index in Ureje dam during the dry season.

\begin{tabular}{|c|c|c|c|c|c|c|c|c|}
\hline$S / N$ & Parameters & Sn & Agencies & Aver. Value & $\mathrm{K}$ & $W_{i}$ & $Q_{i}$ & $Q_{i} W_{i}$ \\
\hline 1 & Temp & 40 & WHO & 27.44 & 0.0130537 & 0.000326343 & 68.6 & 0.022387096 \\
\hline 2 & $\mathrm{pH}$ & 8.5 & ICMB/BIS & 8.11 & 0.0130537 & 0.001535729 & 6.058823529 & 0.009304713 \\
\hline 3 & $\mathrm{DO}_{2}$ & 5 & ICMB/BIS & 6.86 & 0.0130537 & 0.00261074 & 80.625 & 0.210490913 \\
\hline 4 & BOD & 5 & ICMB/BIS & 7.46 & 0.0130537 & 0.00261074 & 149.2 & 0.389522408 \\
\hline 5 & COD & 10 & WHO & 11.8 & 0.0130537 & 0.00130537 & 118 & 0.15403366 \\
\hline 6 & TOT. ALK. & 120 & ICMB & 53.6 & 0.0130537 & 0.000108781 & 44.66666667 & 0.004858877 \\
\hline 7 & TURBIDITY & 5 & WHO & 2.78 & 0.0130537 & 0.00261074 & 55.6 & 0.145157144 \\
\hline 8 & TOT. HAR. & 300 & ICMB/BIS & 97.05 & 0.0130537 & $4.35123 \mathrm{E}-05$ & 32.35 & 0.001407624 \\
\hline 9 & TSS & 25 & NESREA & 7.72 & 0.0130537 & 0.000522148 & 30.88 & 0.01612393 \\
\hline 10 & TDS & 500 & ICMB/BIS & 87.26 & 0.0130537 & $2.61074 \mathrm{E}-05$ & 17.452 & 0.000455626 \\
\hline 11 & TS & & & 94.97 & 0.0130537 & & & \\
\hline 12 & COLOUR & 5 & BIS & 5.42 & 0.0130537 & 0.00261074 & 108.4 & 0.283004216 \\
\hline 13 & CONDUCT. & 300 & $\mathrm{ICMB}$ & 202.56 & 0.0130537 & $4.35123 \mathrm{E}-05$ & 67.52 & 0.002937953 \\
\hline 14 & $\mathrm{NO}_{3}$ & 45 & ICMB/BIS & 0.143 & 0.0130537 & 0.000290082 & 0.317777778 & $9.21817 \mathrm{E}-05$ \\
\hline 15 & $\mathrm{NO}_{2}$ & 0.2 & NIS & 0.033 & 0.0130537 & 0.0652685 & 16.5 & 1.07693025 \\
\hline 16 & $\mathrm{PO}_{4}$ & 0.1 & USEPA & 0.024 & 0.0130537 & 0.130537 & 24 & 3.132888 \\
\hline 17 & $\mathrm{Na}$ & 200 & NIS & 10.74 & 0.0130537 & $6.52685 \mathrm{E}-05$ & 5.37 & 0.000350492 \\
\hline 18 & $\mathrm{Ca}$ & 75 & WHO & 23.18 & 0.0130537 & 0.000174049 & 30.90666667 & 0.005379285 \\
\hline 19 & $\mathrm{~K}$ & 10 & WHO & 11.6 & 0.0130537 & 0.00130537 & 116 & 0.15142292 \\
\hline 20 & $\mathrm{Fe}$ & 0.1 & WHO & 0.31 & 0.0130537 & 0.130537 & 310 & 40.46647 \\
\hline 21 & $\mathrm{Mn}$ & 0.1 & BIS & 0.19 & 0.0130537 & 0.130537 & 190 & 24.80203 \\
\hline 22 & $\mathrm{Zn}$ & 3 & NIS & 0.12 & 0.0130537 & 0.004351233 & 4 & 0.017404933 \\
\hline 23 & $\mathrm{Mg}$ & 30 & BIS & 4.21 & 0.0130537 & 0.000435123 & 14.03333333 & 0.006106231 \\
\hline 24 & $\mathrm{Cu}$ & 0.05 & BIS & 0.12 & 0.0130537 & 0.261074 & 240 & 62.65776 \\
\hline \multirow[t]{3}{*}{25} & $\mathrm{~Pb}$ & 0.05 & WHO & 0.001 & 0.0130537 & 0.261074 & 2 & 0.522148 \\
\hline & & & & & $\sum W_{i}=$ & 1.00000309 & $\sum Q_{i} W_{i}=$ & 134.0786665 \\
\hline & & & & & & $\sum Q_{i} W_{i} / \sum W_{i}$ & $=134.08$ & \\
\hline
\end{tabular}

Table 6. Water quality index in Ureje dam during the rainy season.

\begin{tabular}{ccccccccc}
\hline S/N & Parameters & Sn & Agencies & Aver. Value & K & Wi & Qi & QiWi \\
\hline 1 & Temp & 40 & WHO & 25.66 & 0.0130537 & 0.000326343 & 64.15 & 0.020934871 \\
2 & $\mathrm{pH}$ & 8.5 & ICMB/BIS & 8.72 & 0.0130537 & 0.001535729 & 13.23529412 & 0.02032583 \\
3 & $\mathrm{DO}_{2}$ & 5 & ICMB/BIS & 7.65 & 0.0130537 & 0.00261074 & 72.39583333 & 0.189006698 \\
4 & BOD & 5 & ICMB/BIS & 6.32 & 0.0130537 & 0.00261074 & 126.4 & 0.329997536 \\
5 & COD & 10 & WHO & 9.53 & 0.0130537 & 0.00130537 & 95.3 & 0.124401761 \\
6 & TOT. ALK. & 120 & ICMB & 47.41 & 0.0130537 & 0.000108781 & 39.50833333 & 0.004297749 \\
7 & TURBIDITY & 5 & WHO & 3.43 & 0.0130537 & 0.00261074 & 68.6 & 0.179096764 \\
8 & TOT. HAR. & 300 & ICMB/BIS & 86.32 & 0.0130537 & $4.35123 \mathrm{E}-05$ & 28.77333333 & 0.001251995 \\
\hline
\end{tabular}




\section{Continued}

\begin{tabular}{|c|c|c|c|c|c|c|c|c|}
\hline 9 & TSS & 25 & NESREA & 6.98 & 0.0130537 & 0.000522148 & 27.92 & 0.014578372 \\
\hline 10 & TDS & 500 & ICMB/BIS & 92.62 & 0.0130537 & $2.61074 \mathrm{E}-05$ & 18.524 & 0.000483613 \\
\hline 11 & TS & & & 99.53 & 0.0130537 & & & \\
\hline 12 & COLOUR & 5 & BIS & 7.29 & 0.0130537 & 0.00261074 & 145.8 & 0.380645892 \\
\hline 13 & CONDUCT. & 300 & ICMB & 212.65 & 0.0130537 & $4.35123 \mathrm{E}-05$ & 70.88333333 & 0.003084299 \\
\hline 14 & $\mathrm{NO}_{3}$ & 45 & ICMB/BIS & 0.2 & 0.0130537 & 0.000290082 & 0.444444444 & 0.000128925 \\
\hline 15 & $\mathrm{NO}_{2}$ & 0.2 & NIS & 0 & 0.0130537 & 0.0652685 & 0 & 0 \\
\hline 16 & $\mathrm{PO}_{4}$ & 0.1 & USEPA & 0 & 0.0130537 & 0.130537 & 0 & 0 \\
\hline 17 & $\mathrm{Na}$ & 200 & NIS & 22.39 & 0.0130537 & $6.52685 \mathrm{E}-05$ & 11.195 & 0.000730681 \\
\hline 18 & $\mathrm{Ca}$ & 75 & WHO & 29.47 & 0.0130537 & 0.000174049 & 39.29333333 & 0.006838978 \\
\hline 19 & $\mathrm{~K}$ & 10 & WHO & 17.5 & 0.0130537 & 0.00130537 & 175 & 0.22843975 \\
\hline 20 & $\mathrm{Fe}$ & 0.1 & WHO & 0.2 & 0.0130537 & 0.130537 & 200 & 26.1074 \\
\hline 21 & $\mathrm{Mn}$ & 0.1 & BIS & 0.12 & 0.0130537 & 0.130537 & 120 & 15.66444 \\
\hline 22 & $\mathrm{Zn}$ & 3 & NIS & 0.3 & 0.0130537 & 0.004351233 & 10 & 0.043512333 \\
\hline 23 & $\mathrm{Mg}$ & 30 & BIS & 4.96 & 0.0130537 & 0.000435123 & 16.53333333 & 0.007194039 \\
\hline 24 & $\mathrm{Cu}$ & 0.05 & BIS & 0.16 & 0.0130537 & 0.261074 & 320 & 83.54368 \\
\hline \multirow[t]{3}{*}{25} & $\mathrm{~Pb}$ & 0.05 & WHO & 0.001 & 0.0130537 & 0.261074 & 2 & 0.522148 \\
\hline & & & & & $\sum W_{i}=$ & 1.00000309 & $\sum Q_{i} W_{i}=$ & 127.3926181 \\
\hline & & & & & & $\sum Q_{i} W_{i} / \sum W_{i}$ & $=127.39$ & \\
\hline
\end{tabular}

Table 7. Water quality index in Ero dam during the dry season.

\begin{tabular}{|c|c|c|c|c|c|c|c|c|}
\hline S/N & Parameters & Sn & Agencies & Aver. Value & $\mathrm{K}$ & Wi & Qi & QiWi \\
\hline 1 & Temp & 40 & WHO & 26.75 & 0.0130537 & 0.000326343 & 66.875 & 0.021824155 \\
\hline 2 & $\mathrm{pH}$ & 8.5 & ICMB/BIS & 9.05 & 0.0130537 & 0.001535729 & 17.11764706 & 0.026288074 \\
\hline 3 & $\mathrm{DO}_{2}$ & 5 & ICMB/BIS & 8.85 & 0.0130537 & 0.00261074 & 59.89583333 & 0.156372448 \\
\hline 4 & BOD & 5 & ICMB/BIS & 5.21 & 0.0130537 & 0.00261074 & 104.2 & 0.272039108 \\
\hline 5 & COD & 10 & WHO & 8.62 & 0.0130537 & 0.00130537 & 86.2 & 0.112522894 \\
\hline 6 & TOT. ALK. & 120 & ICMB & 46.1 & 0.0130537 & 0.000108781 & 38.41666667 & 0.004178997 \\
\hline 7 & TURBIDITY & 5 & WHO & 2.76 & 0.0130537 & 0.00261074 & 55.2 & 0.144112848 \\
\hline 8 & TOT. HAR. & 300 & ICMB/BIS & 76.07 & 0.0130537 & $4.35123 \mathrm{E}-05$ & 25.35666667 & 0.001103328 \\
\hline 9 & TSS & 25 & NESREA & 6.15 & 0.0130537 & 0.000522148 & 24.6 & 0.012844841 \\
\hline 10 & TDS & 500 & ICMB/BIS & 52.17 & 0.0130537 & $2.61074 \mathrm{E}-05$ & 10.434 & 0.000272405 \\
\hline 11 & TS & & & 58.34 & 0.0130537 & & & \\
\hline 12 & COLOUR & 5 & BIS & 5 & 0.0130537 & 0.00261074 & 100 & 0.261074 \\
\hline 13 & CONDUCT. & 300 & ICMB & 124.93 & 0.0130537 & 4.35123E-05 & 41.64333333 & 0.001811999 \\
\hline 14 & $\mathrm{NO}_{3}$ & 45 & ICMB/BIS & 0.187 & 0.0130537 & 0.000290082 & 0.415555556 & 0.000120545 \\
\hline 15 & $\mathrm{NO}_{2}$ & 0.2 & NIS & 0.002 & 0.0130537 & 0.0652685 & 1 & 0.0652685 \\
\hline 16 & $\mathrm{PO}_{4}$ & 0.1 & USEPA & 0.002 & 0.0130537 & 0.130537 & 2 & 0.261074 \\
\hline 17 & $\mathrm{Na}$ & 200 & NIS & 40.22 & 0.0130537 & $6.52685 \mathrm{E}-05$ & 20.11 & 0.00131255 \\
\hline 18 & $\mathrm{Ca}$ & 75 & WHO & 53.12 & 0.0130537 & 0.000174049 & 70.82666667 & 0.012327334 \\
\hline
\end{tabular}




\section{Continued}

\begin{tabular}{ccccccccc}
\hline 19 & $\mathrm{~K}$ & 10 & WHO & 31.57 & 0.0130537 & 0.00130537 & 315.7 & 0.412105309 \\
20 & $\mathrm{Fe}$ & 0.1 & WHO & 0.06 & 0.0130537 & 0.130537 & 60 & 7.83222 \\
21 & $\mathrm{Mn}$ & 0.1 & BIS & 0.096 & 0.0130537 & 0.130537 & 96 & 12.531552 \\
22 & $\mathrm{Zn}$ & 3 & $\mathrm{NIS}$ & 0.101 & 0.0130537 & 0.004351233 & 3.366666667 & 0.014649152 \\
23 & $\mathrm{Mg}$ & 30 & BIS & 2.25 & 0.0130537 & 0.000435123 & 7.5 & 0.003263425 \\
24 & $\mathrm{Cu}$ & 0.05 & BIS & 0.03 & 0.0130537 & 0.261074 & 60 & 15.66444 \\
25 & $\mathrm{~Pb}$ & 0.05 & WHO & 0.0031 & 0.0130537 & 0.261074 & 6.2 & 1.6186588 \\
& & & & & $\sum W_{i}=$ & 1.00000309 & $\sum Q_{i} W_{i}=$ & 39.43143671 \\
& & & & & & & & \\
\end{tabular}

Table 8. Water quality index in Ero dam during the rainy season.

\begin{tabular}{|c|c|c|c|c|c|c|c|c|}
\hline S/N & Parameters & Sn & Agencies & Aver. Value & $\mathrm{K}$ & $\mathrm{Wi}$ & Qi & QiWi \\
\hline 1 & Temp & 40 & WHO & 26.14 & 0.0130537 & 0.000326343 & 65.35 & 0.021326482 \\
\hline 2 & $\mathrm{pH}$ & 8.5 & ICMB/BIS & 9.07 & 0.0130537 & 0.001535729 & 17.35294118 & 0.026649422 \\
\hline 3 & $\mathrm{DO}_{2}$ & 5 & ICMB/BIS & 10.07 & 0.0130537 & 0.00261074 & 47.1875 & 0.123194294 \\
\hline 4 & BOD & 5 & ICMB/BIS & 5.54 & 0.0130537 & 0.00261074 & 110.8 & 0.289269992 \\
\hline 5 & $\mathrm{COD}$ & 10 & WHO & 9.13 & 0.0130537 & 0.00130537 & 91.3 & 0.119180281 \\
\hline 6 & TOT. ALK. & 120 & ICMB & 36.61 & 0.0130537 & 0.000108781 & 30.50833333 & 0.003318722 \\
\hline 7 & TURBIDITY & 5 & WHO & 2.39 & 0.0130537 & 0.00261074 & 47.8 & 0.124793372 \\
\hline 8 & TOT. HAR. & 300 & ICMB/BIS & 75.34 & 0.0130537 & $4.35123 \mathrm{E}-05$ & 25.11333333 & 0.00109274 \\
\hline 9 & TSS & 25 & NESREA & 6.35 & 0.0130537 & 0.000522148 & 25.4 & 0.013262559 \\
\hline 10 & TDS & 500 & ICMB/BIS & 57.42 & 0.0130537 & $2.61074 \mathrm{E}-05$ & 11.484 & 0.000299817 \\
\hline 11 & TS & & & 63.77 & 0.0130537 & & & \\
\hline 12 & COLOUR & 5 & BIS & 5 & 0.0130537 & 0.00261074 & 100 & 0.261074 \\
\hline 13 & CONDUCT. & 300 & ICMB & 129.67 & 0.0130537 & $4.35123 \mathrm{E}-05$ & 43.22333333 & 0.001880748 \\
\hline 14 & $\mathrm{NO}_{3}$ & 45 & ICMB/BIS & 0.56 & 0.0130537 & 0.000290082 & 1.244444444 & 0.000360991 \\
\hline 15 & $\mathrm{NO}_{2}$ & 0.2 & NIS & 0 & 0.0130537 & 0.0652685 & 0 & 0 \\
\hline 16 & $\mathrm{PO}_{4}$ & 0.1 & USEPA & 0 & 0.0130537 & 0.130537 & 0 & 0 \\
\hline 17 & $\mathrm{Na}$ & 200 & NIS & 53.9 & 0.0130537 & $6.52685 \mathrm{E}-05$ & 26.95 & 0.001758986 \\
\hline 18 & $\mathrm{Ca}$ & 75 & WHO & 52.54 & 0.0130537 & 0.000174049 & 70.05333333 & 0.012192736 \\
\hline 19 & $\mathrm{~K}$ & 10 & WHO & 26.11 & 0.0130537 & 0.00130537 & 261.1 & 0.340832107 \\
\hline 20 & $\mathrm{Fe}$ & 0.1 & WHO & 0.081 & 0.0130537 & 0.130537 & 81 & 10.573497 \\
\hline 21 & $\mathrm{Mn}$ & 0.1 & BIS & 0.24 & 0.0130537 & 0.130537 & 240 & 31.32888 \\
\hline 22 & $\mathrm{Zn}$ & 3 & NIS & 0.39 & 0.0130537 & 0.004351233 & 13 & 0.056566033 \\
\hline 23 & $\mathrm{Mg}$ & 30 & BIS & 5.57 & 0.0130537 & 0.000435123 & 18.566666667 & 0.00807879 \\
\hline 24 & $\mathrm{Cu}$ & 0.05 & BIS & 0.069 & 0.0130537 & 0.261074 & 138 & 36.028212 \\
\hline \multirow[t]{3}{*}{25} & $\mathrm{~Pb}$ & 0.05 & WHO & 0.0048 & 0.0130537 & 0.261074 & 9.6 & 2.5063104 \\
\hline & & & & & $\sum W_{i}=$ & 1.00000309 & $\sum Q_{i} W_{i}$ & 81.84203147 \\
\hline & & & & & & $\sum Q_{i} W_{i} / \sum W_{i}$ & $=81.84$ & \\
\hline
\end{tabular}


show the seasonal WQI for Ero dam during dry season and wet season respectively. The WQI in Egbe dam ranges from 118.00 - 164.67 (dry season) with a mean seasonal value of 145.69 and from 89.39 - 161.86 (rainy season) with a mean seasonal value of 120.59. In Ureje dam, WQI ranges from 101.88 - 164.76 (dry season) with mean seasonal value of 134.08 and from 96.61 - 138.36 (rainy season) with a mean seasonal value of 123.39 while in Ero, WQI ranges from 32.28 - 45.13 (dry season) with a mean seasonal value of 39.43 and from 52.12 106.22 (rainy season) with a mean seasonal value of 81.84 .

According to the rating of water status based on WQI by [21] and [22], the WQI of Egbe dam and Ureje dam during the dry season and rainy season indicated poor water quality that is was suitable for fisheries, recreational, industrial and irrigation purposes but unfit for drinking while in Ero dam, the WQI during the dry season indicated good quality water while during the rainy season, the water in the dam was polluted. The grossly polluted nature of Egbe dam is likely due to anthropogenic activities such as bathing, cleaning, fishing, intense farming activities around the dam and washing of farm produce into the dam while that of Ureje is likely due to fishing, leachates from solid wastes like the paper, polythene bags, plastic cups, sachets, straws, cloths and leaves that are scattered on the water and around the bank of the dam in addition to domestic effluents and sewage released directly into the dam from residential buildings around the dam (Plate 1), where it is situated in central part of Ado-Ekiti, the State Capital. In addition, the use of canoes as main transport around the dams' area in Egbe dam and Ero dam contributed to the polluted state of the dams. The impact of various anthropogenic activities was evident in the high BOD level in all the dams since BOD values indicate a possibility of organic pollution effect on the water of the dams. High BOD values obtained indicate maximum oxygen consumption and probably pollution load on the aquatic system [23].

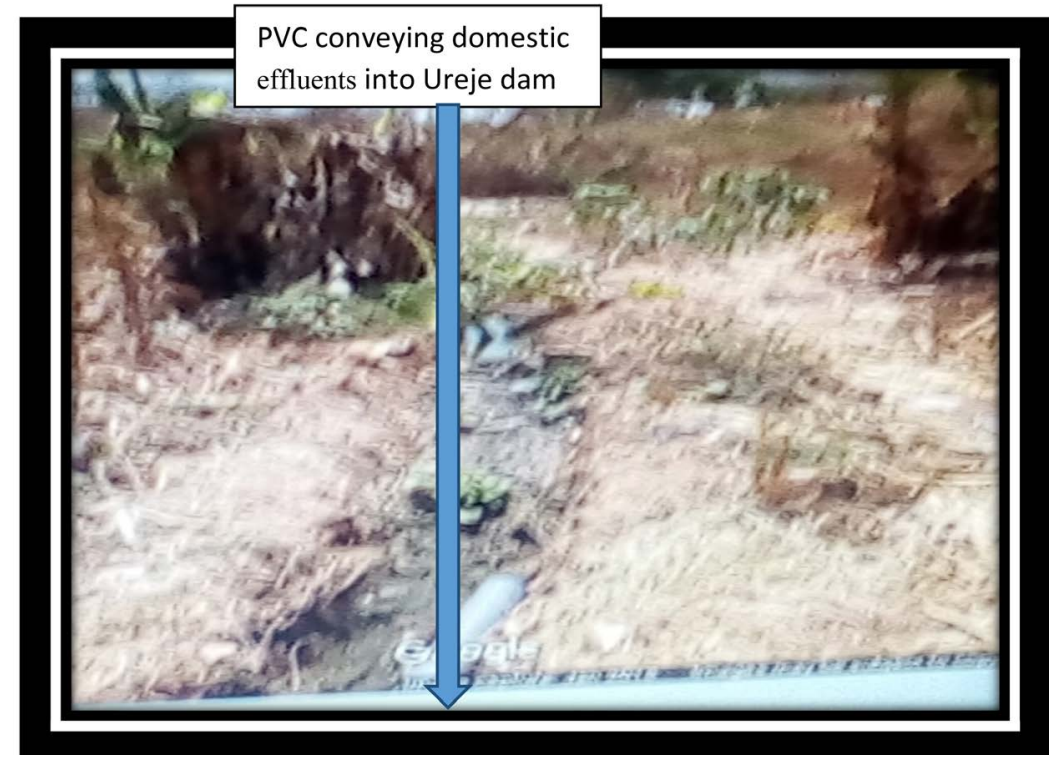

Plate 1. Showing PVC carrying domestic effluents into Ureje dam. 
Figure 2 shows the monthly variations in WQI in the three dams. The variations in WQI in the dams from month to month are likely due the variations in the physico-chemical parameters of the dams each month.

The correlation coefficient values between WQI and the water physico-chemical parameters show that COD, TSS, TDS, Total solids, $\mathrm{NO}_{3}, \mathrm{PO}_{4}, \mathrm{Fe}$ and $\mathrm{Pb}$ were the most affecting factors for computed WQI values in Egbe dam (Table 9) while $\mathrm{BOD}, \mathrm{Mn}$ and $\mathrm{Cu}$ were most affecting parameters for the computed WQI in Ureje dam (Table 10). In Ero dam, temperature, turbidity and Ca values were the most affecting parameters for the computed WQI (Table 11). In this study, there was significant difference at $\mathrm{p} \geq 0.05$ between WQI during dry season and the rainy season in Egbe dam and Ero dam while there was no significant difference at $\mathrm{P} \geq 0.05$ in WQI during the dry season and wet season in Ureje dam. However, the average WQI in Egbe dam and Ureje dam during the rainy season was slightly lower than an average WQI for dry season (Figure 3). Thus, indicating better quality during the rainy season than the dry season in the dams. Similar observation was reported by [24] on Nokoue Lake. This observation indicate that rainfall doesn't have adverse effects on water quality but instead improve environmental water bodies possibly due to the increase in $\mathrm{DO}_{2}$

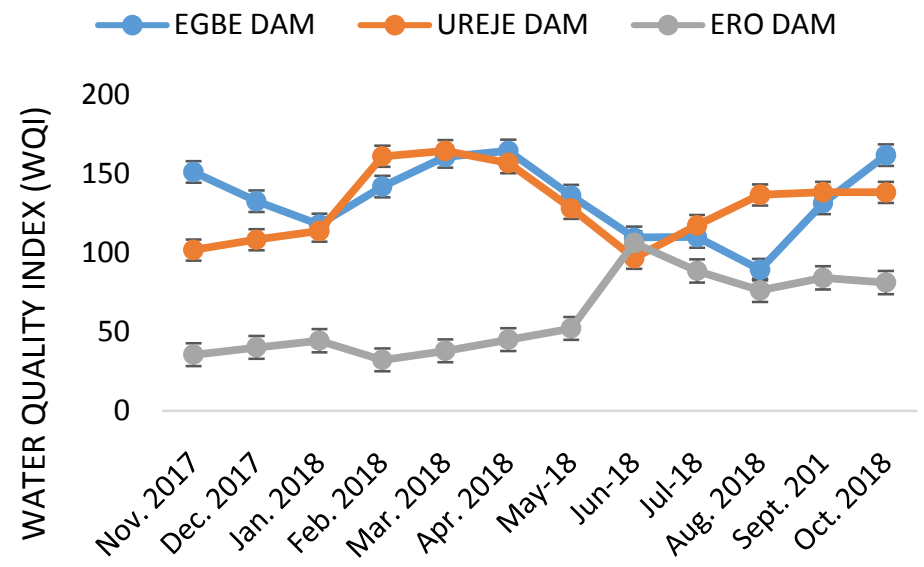

Figure 2. Monthly variations in WQI in the dams.

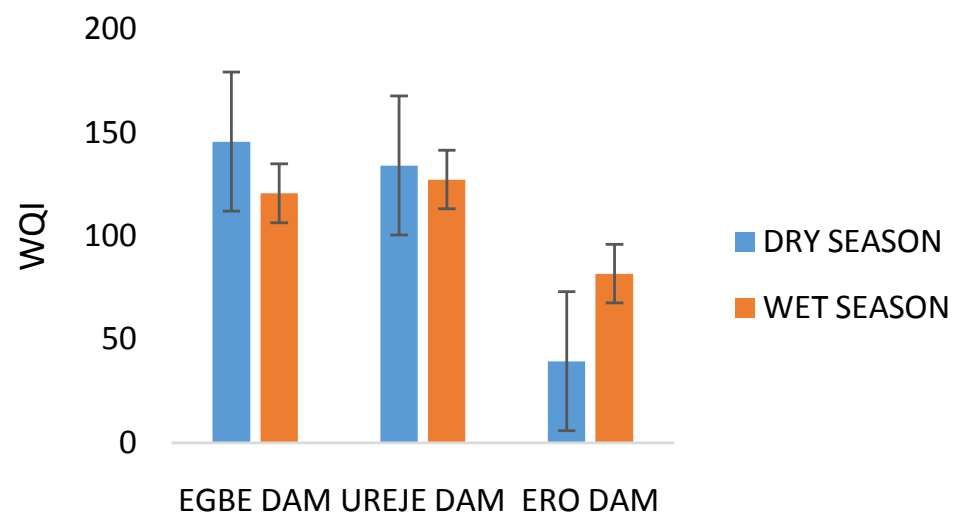

Figure 3. Seasonal variations in values of WQI in the three dams. 


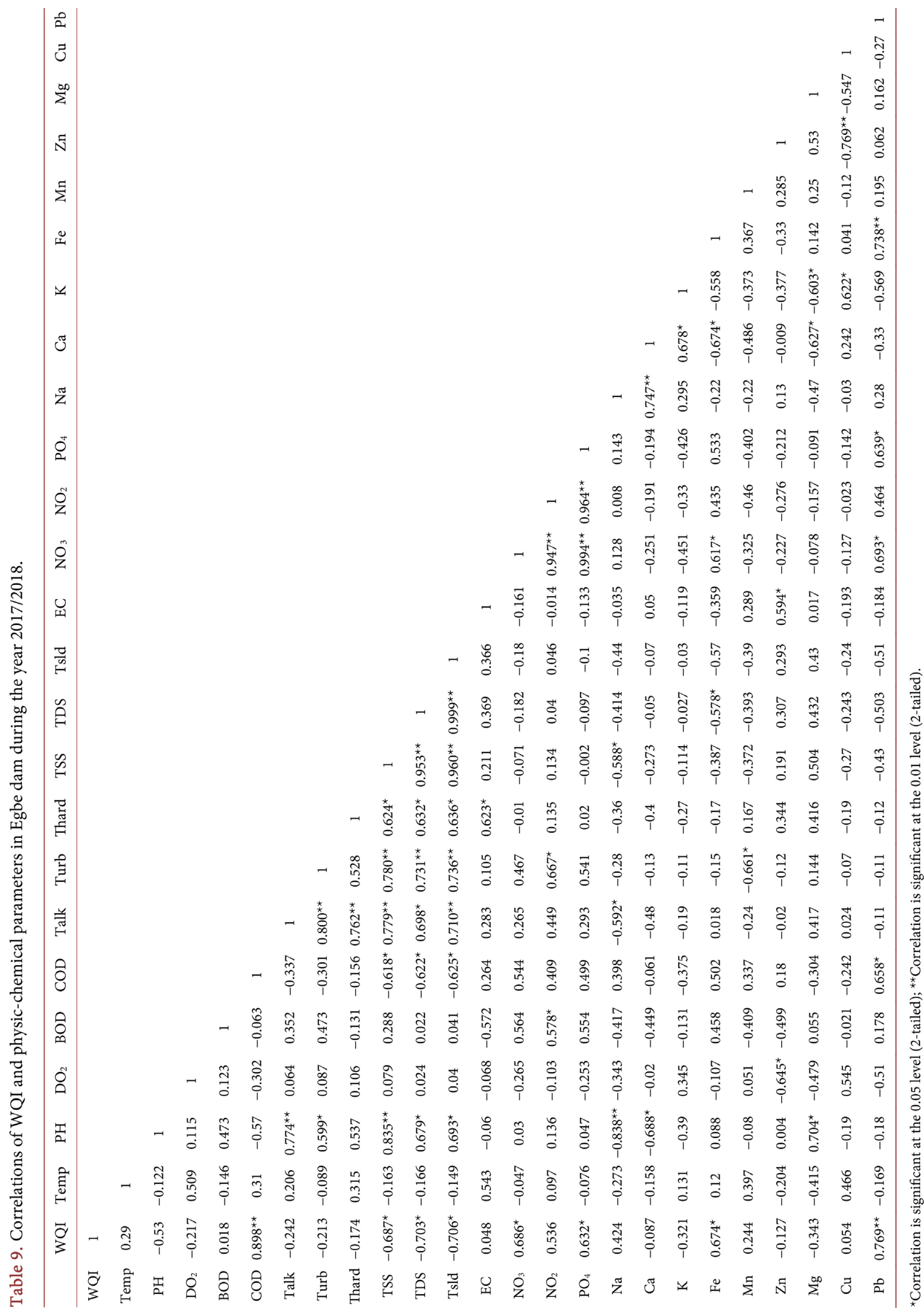




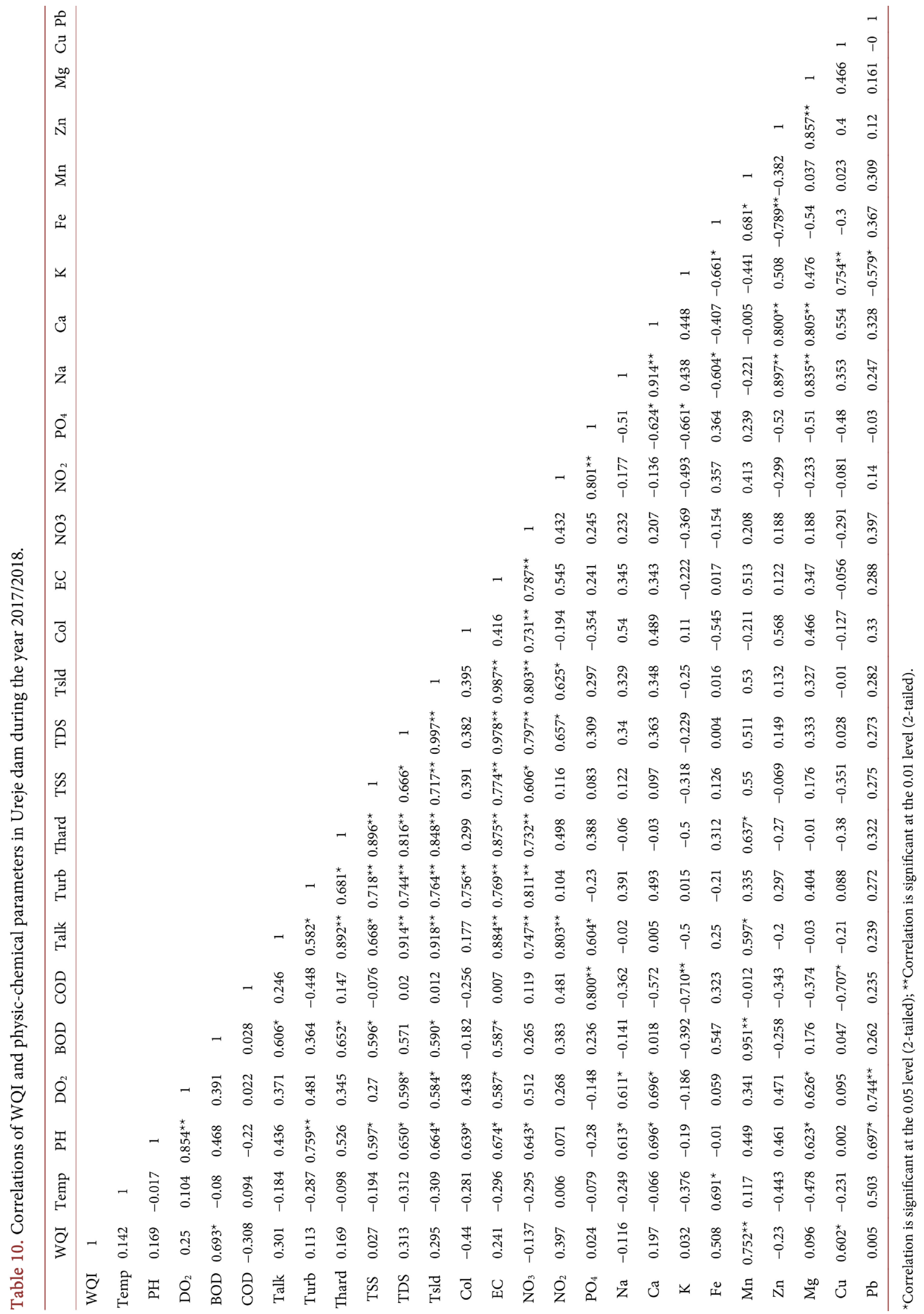




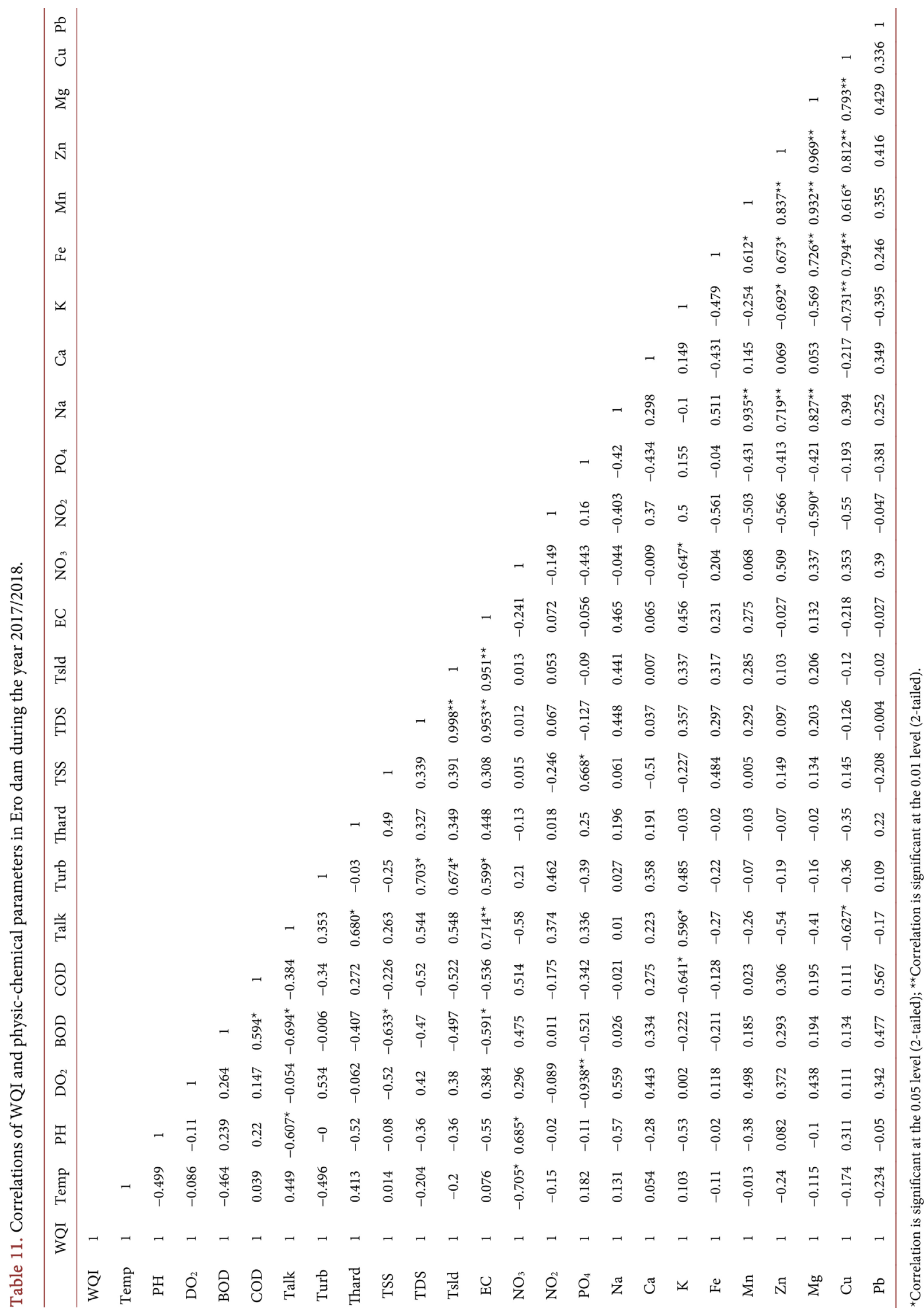


during the rainy season and according to [26], it indicates the absence of dirty runoff entering the dams during the rainy season as the TSS, TDS and total solids levels in all the dams during the rainy seasons were far below the recommended limits. The study thus indicates quite significant seasonal influence of rainfalls on the dam aquatic ecosystems by improving the water quality. This observation is similar to the report of [26], that seasonal variations in precipitation, surface run-off, groundwater flow, interception and abstraction strongly affect lake volume and consequently the concentration of pollutants in a water body.

In Ero dam, the average WQI during the rainy season was significantly higher $(\mathrm{P} \geq 0.05)$ than an average water quality index for dry season (Figure 3$)$. This was brought about by the increase in $\mathrm{Cu}$ concentration during the rainy season which was likely due to influx of $\mathrm{Cu}$ into the dam from the surrounding.

\section{Conclusion}

Conclusively, the application of the WQI is a very helpful tool that enables decision makers to evaluate water quality and compare water quality of two dams. The study revealed that the WQI of Egbe dam, Ureje dam and Ero dam indicated that the water is poor quality and not totally safe for human consumption, and that it needs treatment before consumption. Thus, there is an urgent need for proper management measures and suitable tools to restore the water quality of these dams for a healthy and promising human society.

\section{Conflicts of Interest}

The authors declare no conflicts of interest regarding the publication of this paper.

\section{References}

[1] Chinhanga, J.R. (2010) Impact of Industrial Effluent from an Iron and Steel Company on the Physico-Chemical Quality of Kwekwe River Water in Zimbabwe. International Journal of Engineering, Science and Technology, 2, 29-40. https://doi.org/10.4314/ijest.v2i7.63754

[2] USEPA (2007) Recent Recommended Water Quality Criteria. United States Environmental Protection Agency. http://www.epa.gov/waterscience/criteria/wqcriteria.html

[3] Jindal, R and Sharma, C. (2011) Studies on Water Quality of Sutlej River around Ludhiana with Reference to Physico-Chemical Parameters. Environmental Monitoring and Assessment, 174, 417-425. https://doi.org/10.1007/s10661-010-1466-8

[4] Kazi, T.G., Arain, M.B., Jamali, M.K., Jalbani, N., Afridi, H.I., Sarfraz, R.A., Baiga, J.A. and Shaha, A.Q. (2009) Assessment of Water Quality of Polluted Lake Using Multivariate Statistical Techniques: A Case Study. Ecotoxicology and Environmental Safety, 72, 301-309. https://doi.org/10.1016/j.ecoenv.2008.02.024

[5] Sarkar, S.K., Saha, M., Takada, H., Bhattacharya, A., Mishra, P. and Bhattacharya, B. (2007) Water Quality Management in the Lower Stretch of the River Ganges, East Coast of India: An Approach through Environmental Education. Journal of Cleaner 
Production, 15, 1559-1567. https://doi.org/10.1016/j.jclepro.2006.07.030

[6] Zhou, F., Huang, G.H., Guo, H.C., Zhang, W. and Hao, Z.J. (2007) Spatio-Temporal Patterns and Source Apportionment of Coastal Water Pollution in Eastern Hong Kong. Water Research, 41, 3429-3439. https://doi.org/10.1016/j.watres.2007.04.022

[7] WHO (2012) Guidelines for Drinking-Water Quality. 4th Edition, World Health Organization, Geneva, Switzerland.

[8] Kolawole, O.M., Ajayi, K.T., Olayemi, A.B. and Okoh, A.I. (2011) Assessment of Water Quality in Asa River (Nigeria) and Its Indigenous Clarias gariepinus Fish. International Journal of Environmental Research and Public Health, 8, 4332-4352. https://doi.org/10.3390/ijerph8114332

[9] Ugwa, A.I. and Wakawa, R.J. (2012) Study of Seasonal Physicochemical Parameters in River Usma. American Journal Environment Science, 8, 569-576. https://doi.org/10.3844/ajessp.2012.569.576

[10] Boukari, Y., Bawa, L.M. and Djaneye-Boundjou, G. (1999) Characterization of some Togo Surface Waters. Bulletin of the Chemical Society of Ethiopia, 13, 11-21. https://doi.org/10.4314/bcse.v13i1.21051

[11] Tyagi, S., Sharma, B., Singh, P. and Dobhal, R. (2013) Water Quality Assessment in Terms of Water Quality Index. American Journal of Water Resources, 1, 34-38.

[12] Horton, R.R. (1965) An Index Number System for Rating Water Quality. Journal of the Water Pollution Control Federation, 37, 300-306.

[13] Adebayo, W.O. (1993) Weather and Climate. In: Ebisemiju, F.S., Ed., Ado-Ekiti Region. A Geographical Analysis and Master Plan, Alpha Prints, Lagos.

[14] Jones, H.A. and Hockey, R.D. (1964) The Geology of Part of Southwestern Nigeria. Geological Survey of Nigeria Bulletin, 31, 1-101.

[15] Prakash, D.R., Jadhav, A.S., Chonde, S.G. and Patil, S.G. (2011) Study of Physicochemical and Biological Characteristics of Lakes from Shivaji University Campus, Kolhapur, Maharashtra. Advances in Applied Science Research, 2, 505-519.

[16] Said, K.S.A., Shuhaimi-Othman, M. and Abas Kutty, A. (2012) The Evaluation of Water Quality and Metal Concentrations of Titiwangsa Lake, Selangor, Peninsular Malaysia. Pakistan Journal of Biological Sciences, 15, 459-468. https://doi.org/10.3923/pjbs.2012.459.468

[17] Tichkule, G.C. and Bakare, S.S. (2017) Physico-Chemical Analysis of Two Freshwater Lakes Near Lakhani Dist. Bhandara (M.S.). Asian Journal of Multidisciplinary Studies, 5, 31-35

[18] AOAC (2005) Official Method of Analysis Association of Analytical Chemist. 15th Edition, AOAC, Washington DC, 11-14.

[19] APHA (American Public Health Association) (1995) Standard Methods for the Examination of Water and Waste Water. 18th Edition, APHA, Washington DC.

[20] Dhirendra, M.J., Alok, K. and Namita, A. (2009) Studies on Physicochemical Parameters to Assess the Water Quality of River Ganga for Drinking Purpose in Haridwar District. Rasayan Journal of Chemical, 2, 195-203.

[21] Charterjee, C. and Raziuddin, M. (2002) Determination of Water Quality Index (WQI) of a Degraded River in Asanol Industrial Area, Raniganj, Burdwan, West Bengal. Nature, Environment and Pollution Technology, 1, 181-189.

[22] Swarnalatha, P., Nageswara, R.K., Ramesh Kumar, P.V. and Harikrishna, M. (2007) Water Quality Assessment by Using an Index at Village Level: A Case Stud. Pollution Research, 26, 619-622. 
[23] Sharma, R. and Capoor, A. (2010) Seasonal Variations in Physical, Chemical and Biological Parameters of Lake Water of Patna Bird Sanctuary in Relation to Fish Productivity. World Applied Sciences Journal, 8, 129-132.

[24] Zandagba, J.E.B., Adandedji, F.M., Lokonon, B.E., Chabi, A., Dan, O. and Mama, D. (2017) Application Use of Water Quality Index (WQI) and Multivariate Analysis for Nokoue Lake Water Quality Assessment. American Journal of Environmental Science and Engineering, 1, 117-127.

[25] Afiq, W.M., Khalik, W.M. and Abdullah, M.P. (2012) Seasonal Influence on Water Quality Status of Temenggor Lake, Perak. The Malaysian Journal of Analytical Sciences, 16, 163-171

[26] Khadka, R.B. and Khanal, A.B. (2008) Environmental Management Plan (EMP) for Melamchi Water Supply Project, Nepal. Environmental Monitoring and Assessment, 146, 225-234. https://doi.org/10.1007/s10661-007-0074-8 